\title{
Avaliação Genética de Vacas e Touros da Raça Gir com Base na Produção de Leite em Diferentes Estádios de Lactação
}

\author{
Rui da Silva Verneque ${ }^{1}$, Mario Luiz Martinez ${ }^{1}$, Roberto Luiz Teodoro ${ }^{1}$
}

\begin{abstract}
RESUMO - O objetivo deste trabalho foi avaliar a viabilidade técnica de se usar a produção de leite em parte da lactação, em vez da produção acumulada até 305 dias, para obtenção dos valores genéticos preditos (VG). Foram comparadas as classificações de touros e vacas pelos seus VG para produção de leite até 90(T90), 150(T150) e 210(T210) dias de lactação, em relação à classificação, com base nos VG calculados, usandose a produção até 305(T305) dias. Adotou-se um modelo animal que incluiu os efeitos fixos de rebanho-ano, época, idade da vaca ao parto e grupo genético e os efeitos aleatórios do animal, do meio permanente e do resíduo. Estimativas de herdabilidade e repetibilidade para T90, T150, T210 e T305 foram $0,21 \pm 0,03$ e $0,47 \pm 0,03 ; 0,23 \pm 0,03$ e $0,50 \pm 0,03 ; 0,24 \pm 0,03$ e $0,50 \pm 0,03$; e $0,23 \pm 0,03$ e $0,49 \pm 0,03$, respectivamente. Correlações genéticas entre T305 e as produções parciais T90, T150 e T210 foram 0,92; 0,96; e 0,98, respectivamente. Correlações de ordem entre os $\mathrm{VG}_{305}$ com $\mathrm{VG}_{90}, \mathrm{VG}_{150}$ e $\mathrm{VG}_{210}$ variaram de $0,83 \mathrm{a} 0$,97 e foram menores, quanto mais curto o período de produção e menor o percentual de animais selecionados (descartados), segundo seus VG. Selecionando-se $20 \%$ dos animais de maior $\mathrm{VG}_{305}$, cerca de 65 , 76 e 87\% deles também seriam escolhidos, se fossem usados $\mathrm{VG}_{90}, \mathrm{VG}_{150}$ ou $\mathrm{VG}_{210}$, respectivamente. Descartando-se $20 \%$ dos animais de menor $\mathrm{VG}_{305}$, apenas cerca

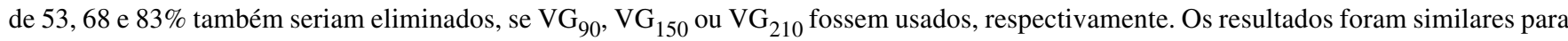
vacas e touros. Concluiu-se que, sob o ponto de vista técnico, o uso da produção de leite em parte da lactação, em curto período, pode resultar em sérios erros na escolha dos melhores ou no descarte dos piores animais do rebanho.
\end{abstract}

Palavras-chave: correlação de ordem, lactação parcial, modelo animal, parâmetros genéticos, raça Gir, seleção

\section{Cow and Sire Genetic Evaluation from Gyr Breed Based on Partial Records of Milk Production}

\begin{abstract}
The objective of this study was to evaluate the viability of using partial records of milk yield instead of the production accumulated at 305 days of lactation to obtain the predicted breeding values (BV). Sires and cows ranked by BV using accumulated milk yield records at 90(T90), 150(T150) and 210(T210) days of lactation were compared with BV estimated by using production up to 305 days (T305). An animal model was used to estimate the animals BV including herd-year, season and age of calving, and genetic group as fixed effects and the animal, permanent environment and residual as random effects. Heritability and repeatability estimates for T90, $\mathrm{T} 150, \mathrm{~T} 210$ and T 305 were $0.21 \pm 0.03$ and $0.47 \pm 0.03 ; 0.23 \pm 0.03$ and $0.50 \pm 0.03 ; 0.24 \pm 0.03$ and $0.50 \pm 0.03 ;$ and $0.23 \pm 0.03$ and $0.49 \pm 0,03$, respectively. Genetic correlations between T305 and T90, T150 and T210 were 0.92, 0.96 and 0.98, respectively. Rank correlations between $\mathrm{BV}_{305}$ and $\mathrm{BV}_{90}, \mathrm{BV}_{150}$ and $\mathrm{BV}_{210}$ ranged from 0.83 to 0.97 and were smaller as production periods and percentage of selected or culled animals based on their BV were reduced. Selecting the top $20 \%$ animals by $\mathrm{BV}_{305}, 65,76$ and $87 \%$ of those animals would be selected if $\mathrm{BV}_{90}, \mathrm{BV}_{150}$ and $\mathrm{BV}_{210}$ were used, respectively. Culling the bottom $20 \%$ animals by $\mathrm{BV}_{305}$, only 53,68 and $83 \%$ would be culled if $\mathrm{BV}_{90}, \mathrm{BV}_{150}$ or $\mathrm{BV}_{210}$ were used, respectively. The results were similar between sires and cows. Therefore, under a technical point of view, the use of partial records of milk yield, in a short period, could result in wrong decisions in the selection of the best or in the culling of the worst animals in the herd.
\end{abstract}

Key Words: animal model, genetic parameter, Gyr breed, partial lactation, rank correlation, selection

\section{Introdução}

Para se estimar a produção de leite na lactação de uma vaca, há necessidade da realização de controle leiteiro. No Brasil, um percentual muito pequeno dos rebanhos participa de controle leiteiro, em decorrência principalmente do seu alto custo. Dessa forma, alguns criadores o realizam apenas nos primeiros meses de lactação e usam essas informações para estimar a produção de leite em toda lactação; a seleção (descarte) dos animais baseia-se na produção obtida em parte da lactação ou na projeção dessa.

Usando-se registros de produção parcial, a avaliação genética pode ser útil, sobretudo, porque permite prever antecipadamente os valores genéticos dos animais. Havendo alta coincidência entre os 
animais selecionados (descartados), usando-se os valores genéticos preditos, com base nos dados de produção na lactação parcial e completa, a decisão sobre seleção (descarte), baseada nos dados de produção na lactação parcial, permitiria reduzir o intervalo de geração, aumentar o ganho genético por geração e diminuir o vício causado pela eliminação de animais antes de se completar a lactação, com economia de alimentos e instalações. Além disso, se este for o caso, não haverá necessidade de se preocupar com o problema de duração da lactação das vacas como indicador de discriminação genética entre animais.

No Brasil, poucos estudos comparando resultados de avaliação genética de vacas e de touros da raça Holandesa, considerando lactações parciais ou produção no dia do controle, têm sido realizados (GENGLER et al., 1995; GADINI et al., 1997). Estes autores constataram alta correlação genética entre produções parciais ou no dia do controle e a produção até 305 dias, especialmente nas produções observadas no terço intermediário da lactação. Não foram encontrados na literatura consultada estudos dessa natureza envolvendo dados com a raça Gir, principalmente comparando a correlação de ordem entre valores genéticos preditos, com base na produção de leite em lactação completa e parcial.

Em outros países, tem-se verificado que a seleção de vacas holandesas pela produção até 305 dias de lactação pode ser substituída pela produção estimada em parte na lactação ou na produção no dia do controle (test day), resultando em resposta anual adicional na produção de leite (TANDON e HARVEY, 1984; FAMULA e VAN VLECK, 1981; WILMINK,1987; SETHI e JAIN, 1993; SWALVE, 1995; e ZAHED et al., 1997). No entanto, verifica-se que as estimativas de correlações genéticas, de ordem e valores diminuem com a redução do período em que o dado de produção é tomado (SETHI e JAIN, 1993; RIBAS et al., 1994).

Assim, o objetivo deste trabalho foi avaliar a viabilidade de se usar a produção de leite acumulada em parte da lactação ou em lactações não-encerradas, em vez da produção até 305 dias, para estimar o valor genético de animais. Para isto, foram comparadas as classificações de touros e vacas, com base nos seus VGs calculados a partir de dados de produção de leite até 90 (T90), 150 (T150), 210 (T210) e 305 (T305) dias de lactação.

\section{Material e Métodos}

Os dados utilizados neste trabalho foram provenientes de animais puros da raça Gir, pertencentes a 47 rebanhos que participam do programa de melhoramento genético da raça, coordenado pela Embrapa Gado de Leite e executado em convênio com a ABCGIL, ou compõem o Arquivo Zootécnico Nacional - Gado de Leite, convênio Embrapa Gado de Leite, ABCZ e ABC. Foram usadas apenas as lactações para as quais se encontram disponíveis os controles leiteiros mensais, necessários para o cálculo da produção nas lactações parciais. Além disso, foram eliminadas as lactações consideradas anormais (doenças ou morte do bezerro ou da vaca antes de encerrar a lactação, venda da vaca etc.) ou lactações em que faltavam as datas dos controles leiteiros ou apresentavam códigos que invalidavam a lactação. Ao final das eliminações, resultaram 8572 lactações de 4805 vacas que pariram entre 1980 e 1997, filhas de 771 touros. Foram usados os dados de produção de leite de cada vaca na data do controle e calculadas as produções acumuladas até 90(T90), 150(T150), 210(T210) e 305(T305) dias, utilizandose o método do intervalo de teste, segundo EVERETT e CARTER (1968). Foram calculados os VGs dos animais considerando-se T90, T150, T210 ou T305 como variável dependente, obtendo-se $\mathrm{VG}_{90}, \mathrm{VG}_{150}$, $\mathrm{VG}_{210}$ e $\mathrm{VG}_{305}$, respectivamente.

As análises foram realizadas usando-se o sistema MTDFREML (BOLDMAN et al., 1995), no qual a avaliação genética se baseia no modelo animal (VAN VLECK, 1992) e usa, para cálculo dos componentes de variância e parâmetros genéticos (herdabilidade, repetibilidade e correlações), o método REML. Foram incluídos no modelo, independentemente da característica, os efeitos fixos de rebanho-ano (261 classes), grupo genético (puro por origem e puro por cruza), época de parto (seca e águas) e a co-variável idade da vaca ao parto, com termos linear e quadrático. Como aleatórios, foram incluídos os efeitos de animal (8214 animais), efeito de meio permanente (4805 vacas) e o resíduo. Foram estimadas as herdabilidades e repetibilidades de cada característica por meio de análise univariada. Estimaram-se as correlações genéticas de T305 com T90, T150 ou T210, em análises bivariadas. Calcularam-se as correlações de ordem entre os VGs obtidos a partir de T90, T150, T210 e T305.

Obtidos os $\mathrm{VG}_{90}, \mathrm{VG}_{150}, \mathrm{VG}_{210}$ e $\mathrm{VG}_{305}$ de cada 
1062 Rev. bras. zootec.

animal, foram consideradas as taxas de seleção ou descarte de $1 ; 5 ; 10 ; 15 ; 20 \%$ ou $100 \%$, de animais, de vacas ou de touros, considerando-se $\mathrm{VG}_{305}$. Foram calculadas as correlações de ordem entre os $\mathrm{VG}_{305}$ com $\mathrm{VG}_{90}, \mathrm{VG}_{150} \mathrm{e} \mathrm{VG}_{210}$, admitindo-se as diferentes situações de seleção ou descarte de animais, vacas ou touros, sob duas condições. A primeira considerou a seleção (descarte) entre os animais avaliados, com confiabilidade da prova maior do que zero ( 8214 animais: 7443 vacas e 771 touros), e a segunda, seleção (descarte) entre animais com confiabilidade da prova superior a 0,60 (2727 animais: 2552 vacas e 175 touros).

Considerando-se os resultados das avaliações genéticas para a produção de leite até $90,150,210$ e 305 dias foram calculados os ganhos genéticos espe$\operatorname{rados}(\Delta \mathrm{G})$, supondo seleção de $10 \%$ dos animais de maior VG, $10 \%$ dos melhores touros e descarte de $20 \%$ das vacas de menor valor genético, admitindose $\Delta \mathrm{G}=\mathrm{REL}_{\mathrm{m}} \mathrm{i} \sigma_{\mathrm{m}}$, em que $\mathrm{REL}_{\mathrm{m}}$ é a confiabilidade média da prova; i, a intensidade de seleção, e $\sigma_{\mathrm{m}}$, o desvio-padrão estimado dos valores genéticos. A confiabilidade (REL) da prova foi calculada como:

$$
R E L=\sqrt{1-\frac{P E V}{\hat{\sigma}_{a}^{2}(1+F A)}}
$$

em que PEV é a variância do erro de predição; FA, o coeficiente de endogamia; e $\hat{\sigma}_{a}^{2}$, estimativa da variância genética aditiva para a característica considerada.

\section{Resultados e Discussão}

Na Tabela 1, são apresentadas as estimativas das médias e os desvios-padrão da produção de leite, do valor genético e da confiabilidade e médias e respectivos erros-padrão da herdabilidade e repetibilidade para T90, T150, T210 e T305. As médias dos valores genéticos preditos e das confiabilidades são apresentadas para todos animais, vacas e touros. Estimativas das herdabilidades e repetibilidades para T90, T150, T210 e T305 foram de mesma magnitude e similares àquelas observadas na literatura para a mesma raça (WILMINK, 1987; SETHI J JAIN, 1993; BALIEIRO, 1998; e VERNEQUE et al., 1998). MADALENA (1988) reportou estimativas de herdabilidade de 0,08 ,

Tabela 1 - Médias e desvios-padrão estimados para produção de leite, valor genético (VG) e confiabilidade (REL) e médias e respectivos erros-padrão da herdabilidade $\left(h^{2}\right)$ e repetibilidade $(r)$ de T90, T150, T210 e T305 para 8214 animais, 7443 vacas e 771 touros

Table 1 - Estimated means and standard deviations for milk production, breeding values (BV) and reliability (REL) and means and standard errors for heritability $\left(h^{2}\right)$ and repeatability $(r)$ for T90, T150, T210 and T305 for 8214 animals, 7443 cows and 771 sires

\begin{tabular}{|c|c|c|c|c|c|}
\hline $\begin{array}{l}\text { Variável } \\
\text { Variable }\end{array}$ & $\begin{array}{l}\text { Média } \\
\text { Mean }\end{array}$ & $\begin{array}{l}\mathrm{VG} \\
B V\end{array}$ & REL & $\mathrm{h}^{2}$ & $\mathrm{r}$ \\
\hline & \multicolumn{5}{|c|}{$\begin{array}{l}\text { Todos animais avaliados }(n=8214) \\
\text { All animals evaluated }(n=8214)\end{array}$} \\
\hline Т90 & $941,7 \pm 332,3$ & $6,1 \pm 50,8$ & $0,47 \pm 0,16$ & $0,21 \pm 0,03$ & $0,47 \pm 0,03$ \\
\hline $\mathrm{T} 150$ & $1462,2 \pm 520,7$ & $10,2 \pm 85,3$ & $0,48 \pm 0,17$ & $0,23 \pm 0,03$ & $0,50 \pm 0,03$ \\
\hline $\mathrm{T} 210$ & $1899,2 \pm 700,4$ & $15,4 \pm 120,6$ & $0,49 \pm 0,17$ & $0,24 \pm 0,03$ & $0,50 \pm 0,03$ \\
\hline \multirow[t]{3}{*}{ T305 } & $2339,2 \pm 953,0$ & $19, \pm 162,2$ & $0,48 \pm 0,17$ & $0,23 \pm 0,03$ & $0,49 \pm 0,03$ \\
\hline & & \multicolumn{2}{|c|}{$\begin{array}{l}\text { Vacas }(\mathrm{n}=7443) \\
\text { Cows }(n=7443)\end{array}$} & \multicolumn{2}{|c|}{$\begin{array}{c}\text { Touros }(\mathrm{n}=771) \\
\text { Sires }(n=771) \\
\end{array}$} \\
\hline & & $\begin{array}{l}\mathrm{VG} \\
B V\end{array}$ & REL & $\begin{array}{l}\mathrm{VG} \\
B V\end{array}$ & REL \\
\hline $\begin{array}{l}\text { T90 } \\
\text { T150 } \\
\text { T210 } \\
\text { T305 }\end{array}$ & & $\begin{array}{c}6,2 \pm 51,1 \\
10,5 \pm 85,9 \\
15,9 \pm 121,5 \\
19,6 \pm 163,4\end{array}$ & $\begin{array}{r}0,47 \pm 0,16 \\
0,49 \pm 0,16 \\
0,50 \pm 0,16 \\
0,50 \pm 0,16\end{array}$ & $\begin{array}{c}4,3 \pm 47,3 \\
7,0 \pm 79,8 \\
10,5 \pm 112,2 \\
13,9 \pm 149,8\end{array}$ & $\begin{array}{c}0,41 \pm 0,20 \\
0,42 \pm 0,20 \\
0,42 \pm 0,20 \\
0,42 \pm 0,20\end{array}$ \\
\hline
\end{tabular}

T90 - Produção de leite até 90 dias.

T150 - Produção de leite até 150 dias.

T210 - Produção de leite até 210 dias.

T305 - Produção de leite até 305 dias.

T90 - Milk production up to 90 days.

T150 - Milk production up to 150 days.

T210 - Milk production up to 210 days.

T305 - Milk production up to 305 days. 
quando estimada ajustando os dados de produção para o efeito de duração da lactação, a 0,44 , quando lactações com duração inferior a 30 dias e produções na lactação menores que $100 \mathrm{~kg}$ foram excluídas e os dados não foram pré-ajustados para a duração da lactação. O autor informa que o ajustamento fenotípico pode reduzir a herdabilidade, quando se remove mais variação genética que ambiente. Neste trabalho, lactações com duração inferior a 90 dias e superior a 600 dias foram excluídas, independente da produção na lactação, mas as estimativas foram obtidas para todas características, considerando-se a mesma amostra, sem ajuste para efeito da duração da lactação. Além disso, a duração média da lactação para os dados considerados foi de 296 dias, existindo percentual pequeno (menos de $5 \%$ ) de vacas com duração de lactação inferior a 150 dias. As estimativas da média e os respectivos desvios-padrão dos valores genéticos, para T90, T150, T210 e T305, variaram de $6,1 \pm 50,8$ a $19,1 \pm 162,2 \mathrm{~kg}$, considerandose todos os 8.214 animais avaliados, de $6,2 \pm 51,1$ a $19,6 \pm 163,4 \mathrm{~kg}$ para 7443 vacas e de $4,3 \pm 47,3$ a $13,9 \pm 149,8 \mathrm{~kg}$ para 771 touros (Tabela 1 ). Observou-se, portanto, incremento em valor absoluto destas estimativas, principalmente do desvio-padrão, com o aumento no número de dias em que as informações de produção na lactação foram tomadas e as estimativas, similares tanto para vacas, quanto para touros, ligeiramente menores para touros.

Correlações genéticas, fenotípicas e de ambiente entre T305 e as produções parciais T90, T150 e T210 variaram respectivamente de 0,92 a 0,$98 ; 0,80$ a 0,95 ; e 0,70 a 0,92 (Tabela 2). Verificam-se maiores estimativas de correlação para produções acumuladas em períodos de lactação mais próximos de 305 dias. Tal comportamento parece independente da confiabilidade da prova do animal ou do erro-padrão das predições, resultado divergente do observado por TANDON e HARVEY (1984).

Os coeficientes de correlação de ordem entre os valores genéticos preditos usando as produções acumuladas T90, T150, T210 e T305 são apresentados na Tabela 3. Os valores acima da diagonal correspondem às estimativas obtidas considerando-se todos animais avaliados, com confiabilidade da prova superior a zero. Considerando-se $\mathrm{VG}_{305}$, as estimativas de correlação foram de 0,$83 ; 0,90$; e 0,96 com $\mathrm{VG}_{90}, \mathrm{VG}_{150}$ e $\mathrm{VG}_{210}$, respectivamente. Valores similares foram obtidos calculando-se as mesmas estimativas, com base em amostras contendo os animais avaliados com confiabilidade da prova superior a 0,60
(Tabela 3, valores abaixo da diagonal). Tal amostra reduziu de 8214 para 2727 animais, sugerindo que as estimativas de correlação foram independentes da confiabilidade da prova.

Nas Tabelas 4 e 5, são apresentados os coeficientes de correlação de ordem entre $V G_{305}$ e os valores genéticos para produções parciais $\mathrm{VG}_{90}, \mathrm{VG}_{150}$ e $\mathrm{VG}_{210}$, considerando-se amostra de todos animais avaliados ou a de touros (os resultados considerando-se apenas as vacas, foram idênticos aos encontrados para a amostra total). Supôs-se que amostras de $1 ; 5 ; 10 ; 15 ; 20 \%$ ou de todos animais avaliados tenham sido consideradas (na seleção ou no descarte), entre aqueles animais com confiabilidade de prova superior a zero (Tabela 4) ou 0,60 (Tabela 5). Notase que, embora as estimativas de correlação para a amostra total sejam altas, selecionando-se $1 \%$ dos animais com maior VG, os coeficientes de correlação foram de 0,50 ; 0,56 ; e 0,72 para $\mathrm{VG}_{305}$ e $\mathrm{VG}_{90}, \mathrm{VG}_{305}$ e $V_{150}$ e $V_{305}$ e $V_{210}$, respectivamente. Selecionando-se $1 \%$ dos melhores touros, as estimativas de correlação foram de 0,$89 ; 0,96$; e 0,96 , respectivamente. Para amostra considerando-se confiabilidade da prova superior a 0,60 , as estimativas de correlação entre $\mathrm{VG}_{305}$ e $\mathrm{VG}_{90}, \mathrm{VG}_{305}$ e $\mathrm{VG}_{150}$ e $\mathrm{VG}_{305}$ e $\mathrm{VG}_{210}$ foram ligeiramente superiores, de 0,$50 ; 0,61$; e 0,75 , considerando-se a seleção de $1 \%$ dos melhores animais igual a unidade, selecionando-se $1 \%$ dos melhores touros. Mantendo-se 20\% dos melhores touros, as estimativas de correlação de ordem entre $V_{305}$ e $\mathrm{VG}_{90}, \mathrm{VG}_{305}$ e $\mathrm{VG}_{150}$ e $\mathrm{VG}_{305}$ e $\mathrm{VG}_{210}$ foram de 0,70 ; 0,80 ; e 0,90 , quando a seleção foi realizada independentemente de confiabilidade da prova dos touros ou de 0,$68 ; 0,72$; e 0,86 , quando se consideraram apenas touros com confiabilidade da prova superior a 0,60 . Descartando-se $20 \%$ das piores vacas, com base em $\mathrm{VG}_{305}$, verificou-se correlação de ordem de 0,$53 ; 0,68$; e 0,83 ou de 0,$78 ; 0,81$; e 0,91 , quando o descarte foi independente de confiabilidade da prova ou considerando-se confiabilidade da prova superior a 0,60 , respectivamente. As estimativas de correlação, para a mesma confiabilidade, aumentaram à medida que maiores percentuais de animais foram considerados, indicando que o tamanho da amostra afeta as estimativas de correlação. Por outro lado, dependendo do tamanho da amostra, verificam-se menores estimativas de correlação entre animais extremos, isto é, entre os melhores ou entre os piores animais. Valores similares para estimativas de correlação são observados quando se comparam os VG dos animais pior classificados. Pode-se observar (Tabela 4), por exemplo, que, 
1064 Rev. bras. zootec.

descartando-se $20 \%$ dos animais de menor $\mathrm{VG}_{305}$, apenas 53,68 ou $83 \%$ desses seriam também descartados, se $\mathrm{VG}_{90}, \mathrm{VG}_{150}$ ou $\mathrm{VG}_{210}$ fossem usados, respectivamente. Estes valores são pouco maiores, se forem considerados para descarte apenas animais com confiabilidade de $\mathrm{VG}_{305}$ maior que 0,60 (Tabela 5). Nota-se, claramente, que, quanto maior o estádio da lactação, maiores as estimativas de correlação com $\mathrm{VG}_{305}$. Estes resultados são esperados e concordantes com os observados na literatura (TANDON e HARVEY, 1984; WILMINK, 1987; SETHI e JAIN, 1993; RIBAS et al., 1994; e WILMINK, 1998) e demonstram que a classificação de vacas e touros é severamente afetada, ao se usar a produção de leite em períodos curtos de produção em relação à produção até 305 dias.

O ganho genético esperado por geração, quando $10 \%$ dos melhores animais foram selecionados, usando-se os $\mathrm{VG}_{90}, \mathrm{VG}_{150}, \mathrm{VG}_{210}$ ou $\mathrm{VG}_{305}$, foram, respectivamente, de 42, 72, 104 e $137 \mathrm{~kg}$. Esses ganhos foram, respectivamente, de 34,59,83,110 kg, se $10 \%$ dos melhores touros forem selecionados, e de $8,4,14,7,21,3$ e $28,6 \mathrm{~kg}$, se forem descartadas $20 \%$ das vacas de menor valor genético. Este resultado é discordante do encontrado por WILMINK (1987), o qual concluiu que a seleção com base na produção de leite entre 90 e 180 dias resultou em ganho genético adicional por geração de $5 \%$ em relação à seleção pela produção até 305 dias de lactação, mas corrobora o observado por TANDON e HARVEY (1984), que obtiveram ganho genético $12 \%$ maior, quando a seleção foi realizada com base na produção até 305 dias em vez da produção até 150 dias. Neste trabalho, o ganho por geração considerando a produção até 305 dias foi, em média, 3,26; 1,9; e 1,32 vezes aquele esperado, se forem usados, respectivamente, T90, T150 ou T210 para predição dos VG. Dessa forma, apesar de a T90 ser obtida com até sete meses de antecedência, parece não recomendável usá-la como critério de seleção ou descarte para esta população, uma vez que a redução em sete meses $(6,4 \%)$ no intervalo de geração, de cerca de 110 meses, não compensaria as perdas no ganho genético esperado. Da mesma forma, a avaliação genética com base em
Tabela 2 - Coeficientes de correlação genética $\left(r_{g}\right)$, fenotípica $\left(r_{p}\right)$ e de ambiente $\left(r_{e}\right)$ entre as produções parciais e a produção até 305 dias

Table 2 - Genetic $\left(r_{g}\right)$, phenotypic $\left(r_{p}\right)$ and environmental correlation coefficients among partial productions and productions up to 305 days

\begin{tabular}{llll}
\hline & T90 & T150 & T210 \\
\hline $\mathrm{r}_{\mathrm{g}}$ & 0,92 & 0,96 & 0,98 \\
$\mathrm{r}_{\mathrm{p}}$ & 0,80 & 0,89 & 0,95 \\
$\mathrm{r}_{\mathrm{e}}$ & 0,70 & 0,83 & 0,92 \\
\hline
\end{tabular}

T90 - Produção de leite até 90 dias.

T210 - Produção de leite até 210 dias.

T150 - Produção de leite até 150 dias.

T305 - Produção de leite até 305 dias.

T90 - Milk production up to 90 days.

T210 - Milk production up to 210 days.

T150 - Milk production up to 150 days.

T305 - Milk production up to 305 days.

Tabela 3 - Coeficientes de correlação de ordem entre $V G_{90}$ $V G_{150}, V_{210}$ e VG $G_{305}$, considerando-se REL de $V G_{305}>$ zero (acima da diagonal, 8214 animais) e REL do $V_{305}>0,60$ (abaixo da diagonal, 2727 animais)

Table 3 - Rank correlation coefficients among $B V_{90}, B V_{150}, B V_{210}$ and $B V_{305}$ considering $R E L$ of $B V_{305}>0$ (upperdiagonal, 8214 animals) and $R E L$ of $B V_{305}>0.60$ (lower diagonal, 2727 animals)

\begin{tabular}{|c|c|c|c|c|}
\hline $\begin{array}{l}\text { Característica } \\
\text { Trait }\end{array}$ & $\begin{array}{l}\mathrm{VG}_{90} \\
B V_{90}\end{array}$ & $\begin{array}{l}\mathrm{VG}_{150} \\
B V_{150}\end{array}$ & $\begin{array}{l}\mathrm{VG}_{210} \\
B V_{210}\end{array}$ & $\begin{array}{r}\mathrm{VG}_{305} \\
B V_{305}\end{array}$ \\
\hline $\mathrm{VG}_{90}$ & - & 0,97 & 0,92 & 0,83 \\
\hline $\begin{array}{l}B V_{90} \\
\mathrm{VG}_{150} \\
B V_{150}\end{array}$ & 0,97 & - & 0,98 & 0,90 \\
\hline $\begin{array}{l}\mathrm{VG}_{210} \\
B V_{210}\end{array}$ & 0,93 & 0,98 & - & 0,96 \\
\hline $\begin{array}{l}\mathrm{VG}_{305}^{210} \\
B V_{305}\end{array}$ & 0,85 & 0,92 & 0,96 & - \\
\hline $\begin{array}{l}V_{V_{90}}-\text { Valor } g \\
V_{G_{150}}-\text { Valor } g \\
V_{150^{210}} \text { - Valor } g \\
V G_{305}-\text { Valor } g \\
B V_{90}-\text { Predicte } \\
B V_{150}-\text { Predicte } \\
B V_{210}-\text { Predicte } \\
B V_{305}-\text { Predicte }\end{array}$ & $\begin{array}{l}\text { nético } \\
\text { nético } \\
\text { nético } \\
\text { nético } \\
\text { breedin } \\
\text { breedin } \\
\text { breedin } \\
\text { breedin }\end{array}$ & $\begin{array}{l}\text { ito usan } \\
\text { ito usan } \\
\text { ito usan } \\
\text { ito usan } \\
\text { ue using } \\
\text { ue using } \\
\text { ue using } \\
\text { ue using }\end{array}$ & $\begin{array}{l}0 . \\
50 . \\
10 . \\
05 .\end{array}$ & \\
\hline
\end{tabular}


VERNEQUE et al.

Tabela 4 - Coeficientes de correlação de ordem entre $V G_{305}$ e $V G_{90}, V_{150}$ e $V G_{210}$, para diferentes percentuais de animais selecionados (descartados) usando-se $V G_{305}$ (REL > 0, 8214 animais)

Table 4 - Rank correlation coefficients among $B V_{305}$ and $B V_{90}, B V_{150}$ and $B V_{210}$ for different percent of selected or culled animals based on $B V_{305}$ (REL $>0,8214$ animals)

\begin{tabular}{|c|c|c|c|c|c|c|c|c|c|c|c|}
\hline \multirow[t]{2}{*}{$\begin{array}{l}\text { Característica } \\
\text { Trait }\end{array}$} & \multicolumn{6}{|c|}{$\begin{array}{l}\text { Porcentagem de animais selecionados } \\
\text { Percent of selected animals }\end{array}$} & \multicolumn{5}{|c|}{$\begin{array}{l}\text { Porcentagem de animais descartados } \\
\text { Percent of culled animals }\end{array}$} \\
\hline & 1 & 5 & 10 & 15 & 20 & 100 & 1 & 5 & 10 & 15 & 20 \\
\hline \multicolumn{12}{|c|}{$\begin{array}{c}\text { Todos animais } \\
\text { All animals }\end{array}$} \\
\hline Т90 & 0,50 & 0,59 & 0,59 & 0,64 & 0,65 & 0,83 & 0,31 & 0,46 & 0,50 & 0,51 & 0,53 \\
\hline $\mathrm{T} 150$ & 0,56 & 0,70 & 0,70 & 0,75 & 0,76 & 0,90 & 0,62 & 0,62 & 0,66 & 0,67 & 0,68 \\
\hline $\mathrm{T} 210$ & 0,72 & 0,82 & 0,84 & 0,87 & 0,87 & 0,96 & 0,82 & 0,82 & 0,82 & 0,82 & 0,83 \\
\hline Animais & 82 & 411 & 821 & 1232 & 1643 & 8214 & 82 & 411 & 821 & 1232 & 1643 \\
\hline \multicolumn{12}{|c|}{$\begin{array}{c}\text { Touros }(\mathrm{n}=771) \\
\text { Sires }(n=771)\end{array}$} \\
\hline T90 & 0,89 & 0,72 & 0,65 & 0,62 & 0,70 & 0,87 & 0,02 & 0,57 & 0,53 & 0,54 & 0,59 \\
\hline T150 & 0,96 & 0,72 & 0,76 & 0,74 & 0,80 & 0,93 & 0,48 & 0,68 & 0,68 & 0,68 & 0,69 \\
\hline T210 & 0,96 & 0,85 & 0,88 & 0,87 & 0,90 & 0,97 & 0,83 & 0,75 & 0,82 & 0,84 & 0,83 \\
\hline $\begin{array}{l}\text { Touros } \\
\text { Sires }\end{array}$ & 8 & 38 & 77 & 115 & 154 & 771 & 8 & 38 & 77 & 115 & 154 \\
\hline
\end{tabular}

Tabela 5 - Coeficientes de correlação de ordem entre $V G_{305}$ com VG $V_{90}, V_{150}$ e $V G_{210}$, para diferentes percentuais de animais selecionados (descartados) usando-se $V_{305}$ ( $R E L>0,60,2727$ animais)

Table 5 - Rank correlation coefficients among $B V_{305}$ with $B V_{90}, B V_{150}$ and $B V_{210}$ for different percent of selected or culled animals based on $B V_{305}$ (REL > 0,60, 2727 animals)

Característica $\quad$ Porcentagem de animais selecionados Porcentagem de animais descartados

Trait Percent of selected animals

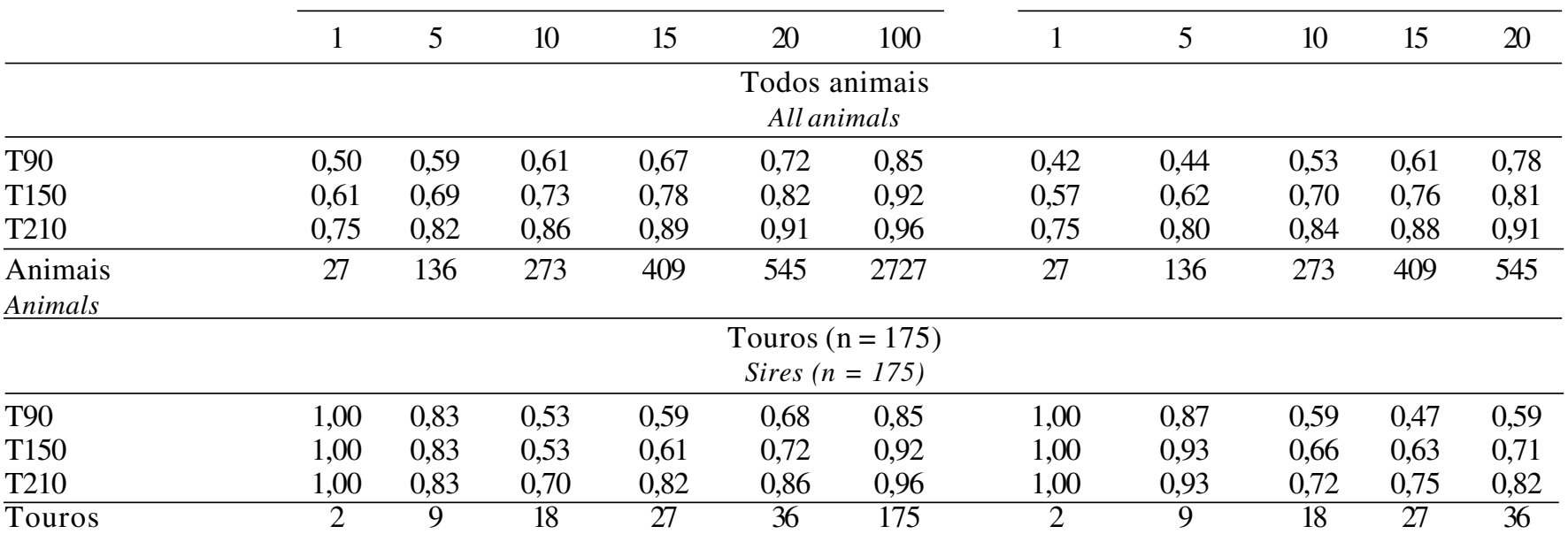

Sires

T150 resultaria também em grandes perdas em ganho genético, com redução no intervalo de geração. Por outro lado, apesar da perda em cerca de $32 \%$ no ganho genético esperado, a produção até 210 dias de lactação poderia ser recomendada especialmente para propósitos de descarte de vacas, pela redução de custos com alimentação de animais geneticamente inferiores.

\section{Conclusões}

Dados de produção de leite de lactações parciais ou não-encerradas são adequados para estimar parâmetros genéticos como herdabilidade e repetibilidade. Todavia, quanto menor o período de produção, menos indicado é o dado para ser usado 
1066 Rev. bras. zootec.

como critério de seleção(descarte) de vacas ou touros.

Apesar de se observarem altas correlações de ordem entre $\mathrm{VG}_{305}$ e os valores de $\mathrm{VG}_{90}, \mathrm{VG}_{150} \mathrm{e}$ $\mathrm{VG}_{210}$, os procedimentos são divergentes, quando se desejam usar os resultados da avaliação genética para seleção(descarte) de animais a serem mantidos ou eliminados dos rebanhos. Assim, considerando-se este conjunto de dados, é pouco recomendável selecionar animais com base na produção de leite em período igual ou inferior a 150 dias, porque resultaria em erros substanciais no descarte de vacas e na seleção de touros. Por outro lado, o uso de $\mathrm{VG}_{210}$ pode ser recomendável especialmente para descarte de vacas pela substancial redução de custo de alimentação de animais geneticamente inferiores.

\section{Referências Bibliográficas}

BALIEIRO, E.S. Estimativas de parâmetros genéticos e tendências genéticas de características reprodutivas e produtivas na raça Gir leiteiro no Brasil. Belo Horizonte, MG: EV-UFMG, 1998. 162p. Tese (Doutoradoem Ciência Animal)-Escola de Veterinária da Universidade Federal de Minas Gerais, 1998.

BOLDMAN, K.G., KRIESE, L.A., VAN VLECK, L.D. et al. 1995. A manual for use of MTDFREML: a set of programs to obtain estimates of variance and covariances. Lincoln: Departament of Agriculture/Agriculture Research Service. 120p.

EVERET, R.W., CARTER, H.W. 1968. Accuracy of test interval method of calculating dairy herd improvement association records. J. Dairy Sci., 51(12):1936-1941.

FAMULA, T.R., VAN VLECK, L.D. 1981. Sire evaluation by only extended partial milk and fat records. J. Dairy Sci., 64(3):484-490.

GADINI, C.H., KEOWN, J.F., VAN VLECK, L.D. Correlações entre produções no dia do controle e em 305 dias de lactação de vacas da raça Holandesa. In: REUNIÃO ANUAL DA SOCIEDADE BRASILEIRA DE ZOOTECNIA, 34, Juiz de Fora, 1997. Anais... Juiz de Fora: SBZ, 1997, p.44-46.

GENGLER, N., KEOWH, J.K., VAN VLECK, L.D. 1995. Various persistency measures and relationships with total, partial and peak milk yields. Rev. Bras. Genet., 18(2):237-243.
MADALENA, F.E. 1988. A note on the effect of variation of lactation length on the efficiency of tropical cattle selection for milk yield. Teoretical Applied Genetics, 76:830-834.

RIBAS, M., PEREZ, B., GUZMAN, G. 1994. Monthly test-day yield and the extension of lactations in Cuba. Cub. J.Agric. Sci., 28(2):125-139.

SETHI, I.C., JAIN, J.P. 1993. Sire evaluation on partial records in dairy cattle. Ind. J. Anim. Sci., 63(8):869-872.

SWALVE, H.H. 1995. Test day models in the analysis on dairy production data - a review. Arch. Tierz. Dummerstorf, 38(6):591-612.

TANDON, P.K., HARVEY, W.R. 1984. Best linear unbiased prediction of sire breeding values from part lactations of daughters. J. Dairy Sci., 67(10):2399-2406.

VAN VLECK, L.D. 1992. Animal model for bull and cow evaluation. In: LARGE DAIRY HERD MANAGEMENT SYMPOSIUM. Gainesville. p.1-31.

VERNEQUE, R.S., MARTINEZ, M.L., TEODORO, R.L Avaliação genética de vacas e touros com base na produção de leite em diferentes estádios da lactação. In: REUNIÃO ANUAL DA SOCIEDADE BRASILEIRA DE ZOOTECNIA, 35, 1998, Botucatu. Anais... Botucatu: SBZ, 1998, p.255-257.

WILMINK, J.B.M. 1987. Efficiency of selection for different cumulative milk, fat and protein yields in first lactation. Lvstck. Prod. Sci., 17: 211-224.

WILMINK, J.B.M. State of art and trends in animal milk recording. In: SIMPÓSIO NACIONAL DA SOCIEDADE BRASILEIRA DE MELHORAMENTO ANIMAL 2, 1998, Uberaba. Anais... Uberaba: SBMA, 1998, p.1-9.

ZAHED, S.M., KHALIL, M.H., SOLIMAN, A.M. 1997. Comparison between efficiency of part-ecompletelactation record in progeny testing Fleckvieh bulls. Egyptian J. Anim. Prod., 34(1):11-26. CD-ROM. CAB Abstracts 01/1996-07/1998.

Recebido em: 30/04/1999 Aceito em: 21/02/2000 